\title{
Optimal Cross-Layer Design of Sampling Rate Adaptation and Network Scheduling for Wireless Networked Control Systems
}

\author{
Jia Bai, Emeka P. Eyisi, Fan Qiu, Yuan Xue, Xenofon D. Koutsoukos \\ Department of Electrical Engineering and Computer Science, Vanderbilt University \\ Email: \{jia.bai, emeka.eyisi, fan.qiu, yuan.xue, xenofon.koutsoukos\}@ vanderbilt.edu
}

\begin{abstract}
Wireless Networked Control Systems (NCS) are increasingly deployed to monitor and control Cyber-Physical Systems (CPS). To achieve and maintain a desirable level of performance, NCS face significant challenges posed by the scarce wireless resource and network dynamics. In this paper, we consider NCS consisting of multiple physical plant and digital controller pairs communicating over a multi-hop wireless network. The control objective is that the plants follow the reference trajectories provided by the controllers. This paper presents a novel optimization formulation for minimizing the tracking error due to (1) discretization and (2) packet delay and loss. The optimization problem maximizes a utility function that characterizes the relationship between the sampling rate and the capability of disturbance rejection of the control system. The constraints come from the wireless network capacity and the delay requirement of the control system. The solution leads to a joint design of sampling rate adaptation and network scheduling, which can be naturally deployed over existing networking systems which have a layered architecture. Based on a passivity-based control framework, we show that the proposed cross-layer design can achieve both stability and performance optimality. Simulation studies conducted in an integrated simulation environment consisting of Matlab/Simulink and ns-2 demonstrate that our algorithm is able to provide agile and stable sampling rate adaptation and achieve optimal NCS performance.
\end{abstract}

Index Terms-wireless networked control system; cross-layer design; sampling rate adaptation; network scheduling

\section{INTRODUCTION}

The integration of physical systems through computing and networking has become a trend, known as Cyber-Physical Systems (CPS). Many real-world CPS such as automotive vehicles and distributed robotics, are monitored and controlled by Networked Control Systems (NCS), where information among sensors, controllers and actuators is exchanged via a communication network. NCS are increasingly deployed over wireless networks, as they provide great convenience in terms of deployment and mobility support [1], [2]. However the stability and the performance of the control system are greatly affected by the limited and dynamic resource availability of the wireless networking environment.

Three major approaches have been investigated in the literature to address the challenges in designing wireless NCS. The first approach, independent of the network protocol design, investigates the design of the control layer with a goal of achieving the desired performance despite of the underlying network uncertainties (e.g., [1], [3]). Alternatively, the network-centric approach focuses on reliable and timely packet deliveries, independent of the control system. Yet without the knowledge and support from the other components of the NCS, these approaches can hardly achieve both stability and optimal performance simultaneously (e.g., [4], [5]). To ensure the stability and optimize the performance of NCS, co-design of the control system and the networking system has been investigated. However, existing work ([6], [7], [8]) either makes simplifying assumptions on the network models or involves too many interactions between the control and the networking systems, which prevents efficient layer abstraction and encapsulation, hindering broader adoption for real-world deployment.

In this paper, we consider NCS consisting of multiple physical plant and digital controller pairs communicating via a multi-hop wireless network, where the plants follow the reference trajectories provided by the controllers. The performance of the NCS is characterized by the tracking errors of the plants which are introduced from two sources: (1) discretization of the controller and the noise disturbance from the operating environment; (2) packet delay and loss caused by the network congestion and dynamics. Both sources of error are related to the sampling rate of the control system. Intuitively, high sampling rates allow frequent state updates and provide NCS with better capability to reduce the effect of environmental disturbances. On the other hand, high sampling rates increase the network load, which increases the possibility of packet loss and delay [9].

We transform the NCS performance objective in terms of tracking error minimization into an optimization problem. The optimization aims at maximizing a utility function that characterizes the relationship between the sampling rate and the capability of disturbance rejection of the control system (i.e., minimizing the discretization-induced tracking error); and the constraints of the sampling rate come from the wireless network capacity and the requirement of sample packet delivery delay. The solution to this optimization problem leads to a cross-layer design of control system sampling rate adaptation and network scheduling, where the sampling rate adaptation determines the bandwidth demands of the network and the scheduling at the media access control layer resolves the location-dependent interference and determines the available resource capacity of each wireless link.

This sample rate optimization problem, however, is nontrivial to solve. The tight coupling of the sampling rate and the required delay of the control system (i.e., the packet delay needs to be less than the sampling time) poses a nonlinear constraint, which has never been addressed in the existing rate optimization solutions ([10], [11]). To solve this problem, we 
present a embedded-loop approach. In the inner loop, a relaxed problem, where the delay bound is fixed and independent of the sampling rate, is solved via dual decomposition. In particular, a double-price scheme is employed to regulate the demand from the sampling rate traffic and the supply of wireless capacity. The capacity price regulates the resource usage at the wireless link level, and the delay price regulates the relationship between the packet delay experienced and the required delay bound at the end-to-end flow level. The control system then adapts its sampling rate based on its utility function so that its net profit, which is the difference between the utility and the cost (product of price and rate), is maximized. The outer loop determines the optimal delay bounds iteratively based on the converged sampling rate from the inner loop. The proposed algorithm naturally leads to a distributed cross-layer implementation.

The main contributions of this paper are summarized as follows. First, we present a new formulation for NCS performance optimization by decoupling its performance metric (tracking error) into two parts - discretization and network effect, which are formulated into the objective and the constraints of an optimization problem respectively. This formulation leads to a cross-layer joint design of sampling rate adaptation and network scheduling which can be easily deployed on existing control systems and networks. We employ a control design approach based on passivity. We formally prove that the stability and the performance optimality of NCS can be simultaneously achieved. Second, we present a distributed algorithm that solves the NCS performance optimization problem and resolves the complex interdependency between the delay and the sampling rate. By introducing a novel Virtual Link Capacity Margin (VLCM) parameter that can be adjusted to control the delay and the rate over a wireless link, our solution does not depend on a specific model of packet arrival process and is suitable for NCS systems where the packet arrivals are not characterized by Poisson process, an assumption routinely made in network delay analysis. Third, our solution is evaluated in an integrated simulation environment that consists of Matlab and ns-2 [12]. Using ns2 - a packet-level network simulator that implements all the details of the network protocol stack, allows highly accurate evaluation of the network effects on the NCS performance, which is impossible by using Matlab/Simulink alone.

The remainder of this paper is organized as follows. Sec. II briefly reviews the related works. In Sec. III, we present the control system model and the wireless network model. In Sec. IV and V, we formulate the problem of optimal rate allocation and present our sampling rate adaptation algorithm. We evaluate the algorithm using the Networked Control System Wind Tunnel (NCSWT) simulation tool in Sec. VI, and conclude the paper in Sec. VII.

\section{RELATED WORK}

There are several existing works on control system sampling rate optimization [10], [11], [13], [14], [8]. This paper considers the interaction of the sampling rate and the end-toend delay experienced by the control systems, which are not addressed by these existing works.

Our work is also related to the work of [15], which compensates for dropout bursts in the network and guarantees the control system stability by reconfiguring the controller or the network. In contrast, our approach assumes a passivitybased control framework which inherently guarantees stability. We focus on the performance optimization with an integrated design of the controller and the network. The framework in [16] obtains the controller's behavior from the aggregate computation of different nodes in the network. As thus, it presents a new NCS paradigm, which is different from the classical NCS model considered in our work. The optimal sampling rate solution presented in [17] focuses on WirelessHart network, which is different from the wireless network model considered in our work.

Utility function has also been used in [18] to capture the relationship between the sampling rate and control performance. However, the optimization formulation in this work leads to an offline solution which can only deal with computing resources with fixed capacity. In comparison, our solution is fully distributed and can handle dynamic wireless resource.

\section{PROBLEM DESCRIPTION}

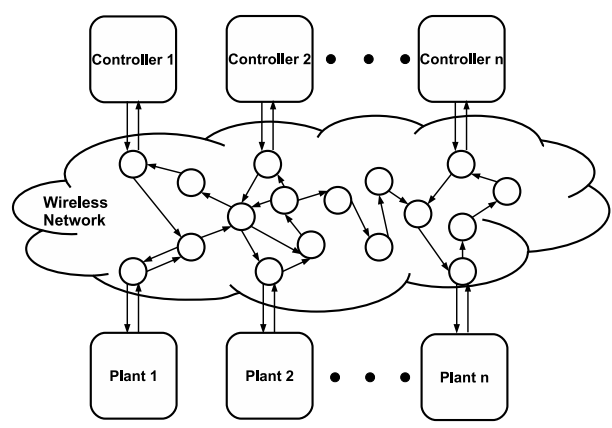

Fig. 1. NCS over multi-hop wireless networks

We consider NCS consisting of multiple plants and digital controllers communicating via a multi-hop wireless network, as shown in Fig. 1. The objective of the control system is that the plants follow the reference trajectories provided by the controllers to complete certain tasks. For example, in a manufacturing factory, a group of robotic operators perform the task of moving objects from one place to another. The network controllers receive desired reference trajectory from the operators and are responsible for ensuring the movement of each robot to track the desired trajectory.

\section{A. Control System Model}

A continuous-time plant is described by

$$
\begin{aligned}
& \dot{x}_{p}(t)=A_{p} x_{p}(t)+B_{p} u_{p}(t)+B_{w} w(t) \\
& y_{p}(t)=C_{p} x_{p}(t)
\end{aligned}
$$


where $x_{p}(t) \in \Re^{n}$ denotes the plant state, $u_{p}(t) \in \Re^{m}$ denotes the control input, $w(t) \in \Re^{m}$ is the disturbance input, and $y_{p}(t) \in \Re^{m}$ is the plant output. $A_{p}, B_{p}$, and $B_{w}$ define the plant state matrices and $C_{p}$ defines the plant output matrix.

The state-space representation of the continuous-time controller is

$$
\begin{aligned}
\dot{x}_{c}(t) & =A_{c} x_{c}(t)+B_{c} u_{c}(t) \\
y_{c}(t) & =C_{c} x_{c}(t)+D_{c} u_{c}(t)
\end{aligned}
$$

where $x_{c}(t) \in \Re^{n}$ denotes the controller state, and $u_{c} \in \Re^{m}$ denotes the error signal, or the difference between the plant output $y_{p}(t) \in \Re^{m}$ and the reference signal input $r(t) \in \Re^{m}$. $A_{c}$ and $B_{c}$ define the controller state matrices, while $C_{c}$ and $D_{c}$ define the controller output matrices. Let the reference signal denote by $r(t)$. The tracking error of the system is

$$
e_{r r}(t)=r(t)-y_{p}(t)
$$

The controller is implemented as a discrete-time control system. We consider sampling instants $t_{k} \in \mathbb{R}, k=0,1, \cdots$, with $t_{k+1}>t_{k}, t_{0}=0$ and we define the sampling interval as $T_{k}=t_{k+1}-t_{k}$. In order to simplify the notations, let $x(k+1)$ represent $x\left(t_{k+1}\right)$, the signal $x(t)$ sampled at time instant $t_{k+1}$.

\section{B. Wireless Network Model}

We model a multi-hop wireless network as a directed graph $G=(V, L)$, where $V$ is the set of wireless nodes in the network. The nodes communicate with each other via directed wireless links $l \in L$. Such a network supports a set of control systems $H$. For each $h \in H$, the traffic from the controller to the plant and the traffic backwards generate two end-to-end flows denoted as $F(h)$. We collect all end-to-end flows in the network into a set $F$. An end-to-end flow $f$ may go through multiple hops in the network and traverse a sequence of links defined by the routing policy. We use set $L(f)$ to represent all the links along the route of flow $f$ and $F(l)$ to denote all the flows that traverse link $l$.

\section{NCS Performance Optimization}

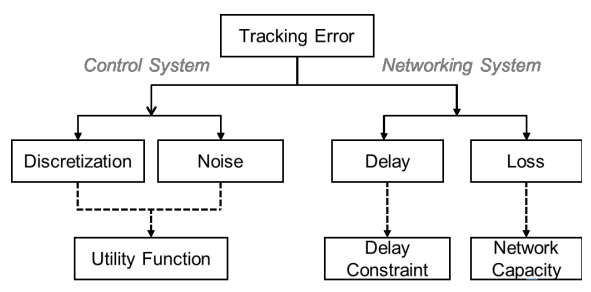

Fig. 2. Decompose tracking error based on its source

The NCS performance can be characterized by the tracking errors of the control systems. The main focus of this paper is to minimize the tracking error of the NCS deployed over the multi-hop wireless network while maintaining certain level of fairness among the plant-controller pairs. As shown in Fig. 2, there are two main sources of error. When a continuoustime control system is discretized, its response to environmental disturbances degrades compared to the response of the idealized continuous system. The level of the degradation depends on the sampling rate, which determines how well the digital controller approximates the continuous controller. High sampling rate allows frequent state updates and thus provides better capability to reduce the effect of environmental disturbances and minimize the tracking error. Packet loss and delay also deteriorate the tracking error. We focus on the congestion-induced packet loss and delay. Network congestion appears when the traffic of the NCS overwhelms the network capacity. While the sampling rate determines the network traffic demand, the network resource management mechanisms such as media access control (MAC) scheduling allocate appropriate capacity to each wireless link.

Optimizing the NCS performance requires the coordination between the control system and the networking system. The control system needs to have the capability to adapt its sampling rate based on the resource utilization information from the network. The networking system should schedule its wireless transmission to meet the resource needs from the control system. This paper studies how to minimize the NCS tracking error via joint sampling rate adaptation and network scheduling. ${ }^{1}$

\section{Optimization Framework For Tracking ERror MINIMIZATION}

In this section, we present the control system design and formulate the problem of NCS tracking error minimization as a sampling rate optimization problem. We first show that our passivity-based control system design is able to ensure system stability with time-varying sampling time. Then we define the optimization objective through a utility function which characterizes the relationship between the sampling rate and the capability of disturbance rejection of the control system (i.e., minimizing the discretization-induced tracking errors). The optimization constraints are based on the wireless network schedulability and the NCS delay requirement.

\section{A. Passivity-based control system - ensuring system stability} with time-varying sampling time

Fig. 3 shows the passivity-based control system architecture. A passive system is defined as a system with bounded output energy such that the system does not produce more energy than what is initially stored. We assume the plant system is passive. A large class of systems can be "passified" by adding local control and filter components [19][20]. The controller $G_{c}(s)$ is designed so that the plant tracks the reference $r(k)$ and is also assumed to be passive. The control architecture uses (1) a discretization approach defined by the Inner Product Equivalent Sampling and Hold (IPESH) transform, which is composed by the Inner Product Equivalent Sampling (IPES) and Zero Order Hold $(\mathrm{ZOH})$ blocks and (2) a bilinear transform $b$ for

\footnotetext{
${ }^{1}$ This paper assumes fixed network routing, which is known a priori.
} 


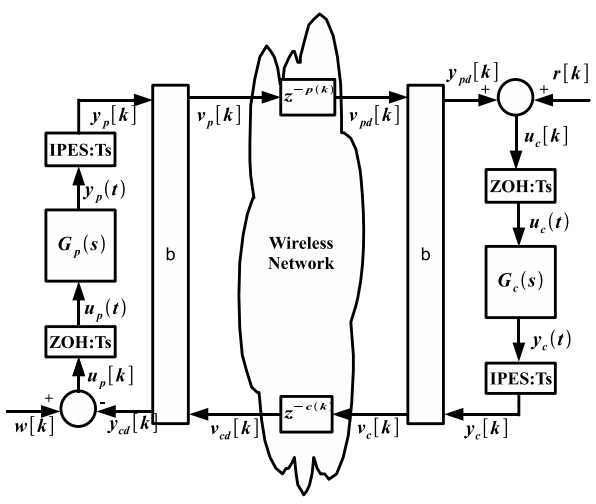

Fig. 3. Passivity Based Control Architecture Over Wireless Networks

converting the control signals into wave variables for communication over a wireless network. These transformations ensure that the NCS are passive and stable in the presence of timevarying delay and packet loss [20], [21]. Next, we show that the NCS are ensured stable with time varying sampling time, which allows us to use sampling rate adaptation.

A passive continuous-time linear time invariant (LTI) system can be converted to a discrete-time passive system at a varying sampling time, $T_{k}$, with the discrete-time state space equations described as

$$
\begin{array}{rc}
x(k+1) & =\Phi_{k} x(k)+\Gamma_{k} u(k) \\
y(k) & =C_{d k} x(k)+D_{d k} u(k)
\end{array}
$$

In [20][22], it is shown that in order to obtain a passive discrete-time equivalent of a LTI passive continuous-time system for a given fixed sampling time $T_{k}$, the IPESH is used to compute the system coefficients, $\Phi_{k}, \Gamma_{k}, C_{d k}$ and $D_{d k}$ to preserve passivity.

Discretization with time-varying sampling time can be performed by applying the IPESH for each resulting sampling time, $T_{k}$, hence ensuring passivity of the discretization at each sampling time and thus the overall passivity of the discretetime system for a given time interval. This implies that the new system coefficients are redefined as $\Phi_{k}=\Phi\left(T_{k}\right), \Gamma_{k}=\Gamma\left(T_{k}\right)$, $C_{d k}=C_{d}\left(T_{k}\right)$ and $D_{d k}=D_{d}\left(T_{k}\right)$. By ensuring the passivity of the discrete-time system, the stability is also ensured.

\section{B. Utility function - modeling error from discretization}

To characterize the impact of the sampling rate on the tracking error, we first introduce a utility function which characterizes the disturbance rejection capability of the discretetime system compared with its continuous-time counterpart.

1) Continuous-time control system: The covariance matrix of the zero-mean white noise process of the continuous-time system can be defined as

$$
E\left[w(t) w^{T}(t+\tau)\right]=Q \delta(\tau)
$$

where $E$ denotes the expected value and $Q$ represents the power spectral density of $w$, or the continuous-time noise covariance matrix. The power spectral density can also be referred to as the "white noise intensity" or mean-square spectral density. The continuous-time state covariance matrix $P_{c}$ can be described by

$$
P_{c}(t)=E\left[x(t) x^{T}(t)\right]
$$

Based on the knowledge of $Q$, the steady state value of the state covariance can be obtained by [23]

$$
A_{c l} P_{c}+P_{c} A_{c l}+B_{w c l} Q B_{w c l}^{T}=0
$$

where the matrices $A_{c l}$ and $B_{w c l}$ denote the closed loop matrices of the continuous-time system, or the coefficients of $x(t)$ and $w(t)$ respectively. From the resulting state covariance matrix, the root mean square of a state can then be determined. The Root-Mean-Square (RMS) of the plant state is equivalent to the standard deviation. For example, assuming a system has only one plant state variable $x_{p}$ and its plant state covariance is $v\left(x_{p}\right)$, the RMS of the plant state is equal to $\sqrt{v\left(x_{p}\right)}$. If a plant has several states, we can use one of the states as long as we compare the same discrete state obtained from the minimal realization of the discretized continuous system.

2) Discrete-time control system: Based on the knowledge of the continuous-time noise covariance matrix $Q$, the discretetime noise covariance matrix $Q_{d}$ can be obtained using the Van Loan's algorithm [24] and can be defined as

$$
Q_{d}=\int_{0}^{T_{f}} \Phi(\tau) B_{w c l} Q B_{w c l}^{T} \Phi^{T}(\tau) d \tau
$$

where $\Phi$ is the closed loop matrix, or the discrete-time state coefficient of $x(k)$, and $B_{w c l}$ denote the closed loop matrix of the continuous-time system, or the coefficient of $x(t)$.

The steady state discrete-time state covariance matrix can then be obtained from the following equation

$$
\Phi P_{d} \Phi^{T}+Q_{d}=P_{d}
$$

From the resulting state covariance matrix, the discrete RMS of the plant state can then be determined in a similar way as the continuous-time case.

3) Utility function formulation: We now define the utility function of a control system as a function of its sampling rate $1 / T_{k}$ using the ratio of RMS between the discrete-time system with its continuous-time counterpart. Thus, the utility function reflects the degradation amount of the system response to the white noise compared to the continuous closed loop system.

$$
U\left(1 / T_{k}\right)=\frac{R M S_{\text {continuous }}}{R M S_{\text {discrete }}\left(T_{k}\right)}
$$

To demonstrate the definition of our utility function, we consider a single-input-single-output (SISO) LTI system without loss of generality. As shown in Fig. 4, its utility function is a strictly concave function of the sampling rate. The concavity of the utility function reflects the marginal return on the control performance when its sampling rate increases. 


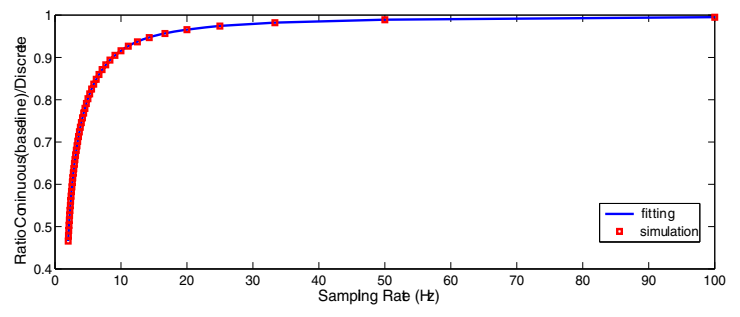

Fig. 4. Example utility function for the control system where the transfer function of the plant is $G_{p}(s)=\frac{1}{J s}$, transfer function of the controller is $G_{c}(s)=\frac{K_{p}+K_{d} s}{s}$, with $J=2.93, K_{d}=32.1$ and $K_{p}=8.2$.

4) Relationship between utility function and tracking error: In a closed-loop continuous-time system, the system response of the plant can be described as

$$
\begin{gathered}
x_{p}(t)=e^{A_{c l} t} x_{p}(0)+e^{A_{c l} t} \int_{0}^{t} e^{-A_{c l} \tau} B_{c l} r(\tau) d \tau \\
+e^{A_{c l} t} \int_{0}^{t} e^{-A_{c l} \tau} B_{w c l} w(\tau) d \tau \\
y_{p}(t)=C_{c l} e^{A_{c l} t} x_{p}(0)+C_{c l} e^{A_{c l} t} \int_{0}^{t} e^{-A_{c l} \tau} B_{c l} r(\tau) d \tau \\
-C_{c l} e^{A_{c l} t} \int_{0}^{t} e^{-A_{c l} \tau} B_{w c l} w(\tau) d \tau
\end{gathered}
$$

Recall that the tracking error of the system $e_{r r}(t)=$ $r(t)-y_{p}(t)$. From (15), the output response of the plant has two main components that contribute towards the tracking error. The first component is the plant response to the reference input $r(t)$, and the other is the plant response to the disturbance input $w(t)$. The passive controller is designed to ensure that the plant's response to the reference input minimizes the tracking error. The system achieves a certain level of disturbance rejection. The contribution of the input disturbance can be characterized by the covariance of the tracking error.

From (15) and the fact that $r(t)$ is not stochastic, we have $E\left[r(t) y^{T}(t)\right]=E\left[r^{T}(t) y(t)\right]=E\left[r(t) r^{T}(t)\right]=0$. The covariance of the tracking error can be described by

$$
C_{e}(t)=E\left[e(t) e^{T}(t)\right]=E\left[y(t) y^{T}(t)\right]
$$

This essentially implies that the covariance of the error is equal to the output covariance. Based on the knowledge of $Q$, the steady state value of the output covariance is [23]

$$
C_{e}=C_{c l} P_{c} C_{c l}^{T}
$$

C. Capacity and delay constraints - bounding error from network

1) Capacity constraint: To limit the effect of packet loss caused by network congestion on the tracking error, we need to restrain the network load within its capacity. Wireless network communication is subject to location dependent interference. Thus the achievable capacity of each wireless link is related to the scheduling algorithm. We adopt the conflict graph concept to model wireless interference [25]. Each vertex in the conflict graph represents a wireless link of the original network and

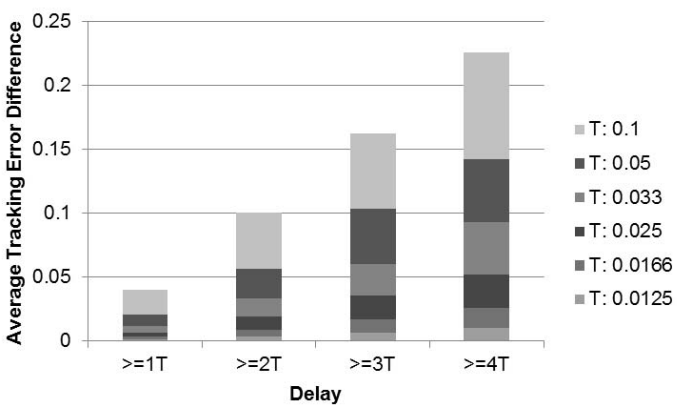

Fig. 5. Impact of Delay On the NCS Average Tracking Error

there is an edge between two vertices if their corresponding wireless links interfere with each other. The communications along wireless links are scheduled on a slotted time basis. In each time slot, one independent $\operatorname{set}^{2} I$ of the conflict graph is selected and only the links corresponding to the vertices in $I$ are allowed to transmit because they are interference free. Let $c_{l}$ be the channel capacity. A $L$-dimension column vector $r^{I}$ is used to represent the capacity vector of $I$, where $r_{l}^{I}=c_{l}$ if $l \in I$, and $r_{l}^{I}=0$ otherwise. We adopt the concept of feasible capacity region $\Lambda$ to model the feasible link capacity allocation [26]. The feasible capacity region is a convex hull, which is defined as $\Lambda:=\sum_{I} \alpha_{I} r^{I}$, where $\sum_{I} \alpha_{I}=$ 1 and $\alpha_{I} \geq 0$. Scheduling essentially determines the capacity allocation $\hat{\boldsymbol{c}}=\left(\hat{c}_{l}, l \in L\right)$ of the links, where $\hat{c}_{l}$ is the average capacity over time based on the scheduling. Obviously, $\hat{c} \in \Lambda$. To limit the packet congestion loss, the aggregated traffic load on any wireless link $l \in L$ should be no more than its achievable capacity $\hat{c}_{l}$.

2) Delay effect on tracking error: To determine the effect of delay on the tracking error, we perform a set of simulation studies using NCSWT [12] over NCS with one pair of plant and controller. Based on the assumption that the discrete plant/controller systems update and process data received only at sampling instants, the delay viewed from the control system's perspective is integral multiples of the sampling interval. We vary the sampling time and manually introduce delays which are integral multiples of the sampling time. Then we evaluate the average tracking error difference, which is the difference between the time-averaged tracking error with delay introduced and the one without any delay.

From the experiment, we observe that when the delay is within one sampling time, the tracking error difference remains zero. Fig. 5 shows the effect of delay on the tracking error difference when it is larger than the sampling time. We observe that the error increases superlinearly when the delay increases beyond one sampling time. Based on the observations, we bound the average end-to-end delay of a control system data flow to its system sampling time.

3) Controlling delay with VLCM: Providing delay assurance is notoriously difficult in wireless networks. The main difficulty comes from the complex interactions between the

\footnotetext{
${ }^{2}$ The independent set of a graph is a set of vertices within which no edge exists between any two vertices.
} 
traffic arrival and departure, which is shaped by the network scheduling. Most of the existing works on delay analysis make explicit assumptions on the packet arrival process (e.g., Poisson arrivals) [27], which do not reflect the NCS traffic characteristics. Here we employ a general method which is not limited to a predefined packet arrival process. In order to regulate the maximum allowable rate $m_{l}$, we introduce a parameter Virtual Link Capacity Margin (VLCM) $\sigma_{l}$ of link $l$ defined by

$$
\sigma_{l}=\hat{c}_{l}-m_{l}, \text { with } m_{l}<\hat{c}_{l}, \forall l \in L
$$

We regard the link delay (i.e., average packet delay along the link) as a function of the $\operatorname{VLCM} \varphi\left(\sigma_{l}\right)$. Then the average delay of flow $f$ is the sum of all link delay along its route.

\section{Optimization Framework}

Recall that each control system is associated with two flows. Let $z_{h}=\frac{\text { sample_size }}{T_{h}}$ be the traffic rate of one flow for the control system $h$, where sample_size is the size of the sample and $T_{h}$ is its sampling time. $T_{h(f)}$ is the sampling time of control system $h$ which flow $f$ is associated with. Thus, the maximum allowable rate satisfies $m_{l} \geq \sum_{h \in H: f \in F(h) \& f \in F(l)} z_{h}$. We overload $U_{h}$ as a function of traffic rate for control system $h$, as defined by Eq. (13). Now we formulate the optimal sampling rate allocation problem as follows:

$$
\begin{aligned}
& W: \max \sum_{h \in H} U_{h}\left(z_{h}\right) \\
& \text { s.t. } \sum_{h \in H: f \in F(h) \cap F(l)} z_{h} \leq \hat{c}_{l}-\sigma_{l}, \forall l \in L \\
& \sum_{l \in L(f)} \varphi\left(\sigma_{l}\right) \leq T_{h(f)}, \forall f \in F \\
& \text { over } \hat{\boldsymbol{c}} \in \Lambda
\end{aligned}
$$

The objective of this nonlinear problem is to maximize the aggregate utility of all control systems in the network. This objective minimizes the tracking error induced by discretization and maintains certain fairness among all the plant-controller pairs [28]. Inequality (20) represents the wireless capacity constraint for each wireless link. Note that the $V L C M \sigma_{l}$ is introduced here to control the link delay. Inequality (22) defines the scheduling feasibility. Inequality (21) is the flow delay constraint where the average flow delay is bounded by the sampling time of its control system. It is important to note that there is a possibility that the optimal solution of the sampling time that minimizes the tracking error may fall below the delay bound. We choose to incorporate this delay bound (21) in our problem formulation for two reasons. First, from Fig. 5, we observe that the tracking error increases super-linearly with respect to delay when the delay goes beyond the sampling time; while the utility only increases sub-linearly with respect to sampling rate. Intuitively, this implies the marginal benefit of increasing the sampling rate is overweighed by the marginal penalty of pushing the delay beyond the sampling time. Based on this intuition, we bound the average delay by the sampling time. On the other hand, without this delay bound constraint, providing a formulation that fully captures the complex interaction among sampling time/rate, delay, delay-introduced error, and discretizationintroduced error will lead to an intractable optimization problem, where identifying a distributed solution is even harder.

\section{Distributed Cross-Layer Algorithm}

\section{A. Solution overview}

Problem $W$ is non-trivial due to the complicated interactions between the $V L C M \mathrm{~s}$, the sampling rate and the end-toend delay. The tight coupling of the sampling rate and the required delay bound of the control system (i.e., the delay needs to be less than the sampling time) poses a nonlinear constraint, which has never been addressed in the existing rate optimization solutions ([10], [11], [29]). To solve this problem, we first relax the delay constraint and consider the optimization problem with a fixed delay requirement. Then we show how to adjust the delay requirement to achieve the optimal solution of the original problem $W$.

\section{B. Cross-layer algorithm with fixed delay}

The optimization framework with a fixed delay requirement can be written as

$$
\begin{array}{ll}
W 1: \quad \max & \sum_{h \in H} U_{h}\left(z_{h}\right) \\
\text { s.t. } & \sum_{h \in H: f \in F(h) \cap F(l)} z_{h} \leq \hat{c}_{l}-\sigma_{l}, \forall l \in L \\
& \sum_{l \in L(f)} \varphi\left(\sigma_{l}\right) \leq D_{h(f)}, \forall f \in F \\
\text { over } & \hat{\boldsymbol{c}} \in \Lambda
\end{array}
$$

where the constraint (21) is replaced by (25), in which $D_{h(f)}$ is the delay requirement of control system $h$.

1) Double-Price Algorithm: Direct solution to $W 1$ requires global coordination of all network components, such as flows and links, which is computationally expensive. We consider its dual decomposition. Let $\boldsymbol{\nu}=\left\{\nu_{l}, l \in L\right\}$ and $\boldsymbol{\mu}=\left\{\mu_{f}, f \in F\right\}$ be the Lagrange multipliers with respect to constraints (24) and (25) respectively. The Lagrangian of $W 1$ is:

$$
\begin{aligned}
\mathcal{L}(\boldsymbol{z}, \boldsymbol{\nu}, \boldsymbol{\sigma}, \boldsymbol{\mu}, \hat{\boldsymbol{c}}) & \\
= & \sum_{h \in H} U_{h}\left(z_{h}\right)-\sum_{l \in L}\left(\nu_{l} \sigma_{l}+\sum_{f \in F(l)} \varphi\left(\sigma_{l}\right) \mu_{f}\right) \\
& -\sum_{h \in H}\left(z_{h} \sum_{l \in L(f) \& f \in F(h)} \nu_{l}\right)+\sum_{f \in F} \mu_{f} D_{h(f)}+\sum_{l \in L} \nu_{l} \hat{c}_{l}
\end{aligned}
$$

The dual of $W 1$ is

$$
\bar{D}(\boldsymbol{\nu}, \boldsymbol{\mu})=\min _{\boldsymbol{\nu} \geq 0, \boldsymbol{\mu} \geq 0} D(\boldsymbol{\nu}, \boldsymbol{\mu})
$$


where

$$
\begin{aligned}
& D(\boldsymbol{\nu}, \boldsymbol{\mu}) \\
& =\max _{\boldsymbol{z}, \boldsymbol{\sigma}, \hat{\boldsymbol{c}}} L(\boldsymbol{z}, \boldsymbol{\nu}, \boldsymbol{\sigma}, \boldsymbol{\mu}, \hat{\boldsymbol{c}}) \\
& =\max _{\boldsymbol{\sigma}}\left\{-\sum_{l \in L}\left(\nu_{l} \sigma_{l}+\sum_{f \in F(l)} \varphi\left(\sigma_{l}\right) \mu_{f}\right)\right\} \\
& +\max _{\boldsymbol{z}}\left\{\sum_{h \in H}\left(U_{h}\left(z_{h}\right)-z_{h} \sum_{l \in L(f) \& f \in F(h)} \nu_{l}\right)\right\} \\
& \quad+\max _{\hat{\boldsymbol{c}}}\left\{\sum_{l \in L} \nu_{l} \hat{c}_{l}\right\}+\sum_{f \in F} \mu_{f} D_{h(f)}
\end{aligned}
$$

The solution $\left(z^{*}, \sigma^{*}, \hat{c}^{*}\right)$ to (28) should satisfy:

$$
\begin{gathered}
z_{h}^{*}=\arg \max _{z_{h}}\left\{\sum_{h \in H}\left(U_{h}\left(z_{h}\right)-z_{h} \sum_{l \in L(f) \& f \in F(h)} \nu_{l}\right)\right\} \\
\sigma_{l}^{*}=\arg \max _{\sigma_{l}}\left\{-\sum_{l \in L}\left(\nu_{l} \sigma_{l}+\sum_{f \in F(l)} \varphi\left(\sigma_{l}\right) \mu_{f}\right)\right\}
\end{gathered}
$$

Here the multiplier $\nu_{l}$ can be seen as the implicit congestion price [29] of link $l$, which represents the cost of delivering a unit of data through link $l$. The multiplier $\mu_{f}$ can be interpreted as the implicit delay price of flow $f$, which represents the cost of imposing a unit of delay on flow $f$. If $\boldsymbol{\nu}$ and $\boldsymbol{\mu}$ are given, we can obtain the maximizers $z_{h}^{*}$ and $\sigma_{l}^{*}$ by taking the derivative with respect to $z_{h}$ and $\sigma_{l}$ respectively.

$$
\begin{gathered}
z_{h}^{*}\left(\kappa_{h}\right)=U_{h}^{\prime-1}\left(\kappa_{h}\right) \cdot \kappa_{h}=\sum_{l \in L(f) \& f \in F(h)} \nu_{l}, \forall h \in H \\
\sigma_{l}^{*}\left(\lambda_{l}, \nu_{l}\right)=\varphi_{l}^{\prime}-1\left(\frac{-\nu_{l}}{\lambda_{l}}\right) . \lambda_{l}=\sum_{f \in F(l)} \mu_{f}, \forall l \in L
\end{gathered}
$$

(32) implies that the optimal sampling rate of a control system $h$ is determined by its price $\kappa_{h}$, which is the aggregated price of the links along its flow routes. (33) implies that the optimal $V L C M$ of a link is relevant to its congestion price $\nu_{l}$ and link margin price $\lambda_{l}$. The intuition is: 1) the congestion price determines the available capacity margin that can be used for $V L C M$ adjustment; and 2) the link margin price implicitly reflects the overall delay requirement (from all of its supporting flow delay requirement) on its $V L C M$. The maximizer $\hat{c}_{l}^{*}$ can be generated from a maximum weight based scheduling policy.

Now $W 1$ is converted into three sub-problems: the sampling rate adaptation problem (29), the VLCM assignment problem (30) and the scheduling problem (31). The link congestion price $\boldsymbol{\nu}$ and the flow delay price $\boldsymbol{\mu}$ can be computed iteratively, from the opposite direction to the gradient $\nabla(L(\boldsymbol{\nu}, \boldsymbol{\mu}))$ [30].
This adaptation approach is called double-price scheme. Based on the information of two price signals, the algorithm iteratively reaches a global optimum. The property of this algorithm is formally characterized in Proposition 1 and Proposition 2.

Proposition 1 There is no duality gap between (23) and (27). For any $\left(\boldsymbol{\nu}^{*}, \boldsymbol{\mu}^{*}\right)$ that minimizes (28), if $\left(\boldsymbol{z}^{*}, \boldsymbol{\sigma}^{*}, \hat{\boldsymbol{c}}^{*}\right)$ solves (29), then $\left(\boldsymbol{z}^{*}, \boldsymbol{\sigma}^{*}, \hat{\boldsymbol{c}}^{*}\right)$ is the unique maximizer of (19).

Proposition 2 If $\|\boldsymbol{\beta}\|_{2}$ and $\|\boldsymbol{\gamma}\|_{2}$ are sufficiently small, starting from any initial values $\boldsymbol{z}(0), \boldsymbol{\sigma}(0), \hat{\boldsymbol{c}}(0)$ and prices $\boldsymbol{\nu}(0) \geq 0, \boldsymbol{\mu}(0) \geq 0$, the cross-layer algorithm converges to the optimal solution $\left(\boldsymbol{z}^{*}, \boldsymbol{\sigma}^{*}, \hat{\boldsymbol{c}}^{*}, \boldsymbol{\nu}^{*}, \boldsymbol{\mu}^{*}\right) .^{3}$

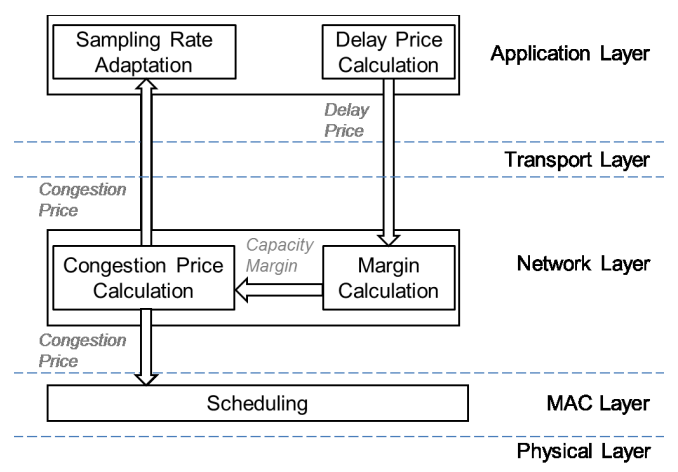

Fig. 6. NCS over multi-hop wireless networks

2) Cross-Layer Rate Allocation Implementation: Our algorithm naturally leads to a cross-layer implementation via joint $V L C M$ assignment, sampling rate adaptation and scheduling, as shown in Fig. 6. Scheduling is performed at the MAC layer. At the network layer, the margin calculation generates the optimal VLCMs for a wireless interface queue; the congestion price calculation provides per-hop congestion price, which reflects the level of congestion at this queue. They can be implemented as part of the queue management mechanism. At the application layer, the per-hop congestion price is aggregated to calculate the sampling rate; the end-to-end delay is measured to calculate the delay price.

Our algorithm implementation only requires the knowledge of the first order derivative of the link delay with respect to the capacity margin $\frac{\partial \varphi\left(\sigma_{l}\right)}{\sigma_{l}}$ based on (33), rather than some statistical characteristics, such as the mean or the variance of the packet arrival rate. The derivative of link delay can be profiled online. According to (31), we need to find a scheduling policy so that the aggregate link weight $\sum_{l \in L} \nu_{l} \hat{c}_{l}$ could be maximized. We achieve this by using a maximum matching based scheduling policy [29].

\section{Delay Bound Tuning}

After obtaining the optimal sampling rate solution to the problem $W 1$ with fixed delay requirement, we now solve the original optimal problem $W$ by determining the optimal delay

\footnotetext{
${ }^{3}$ Due to space needed, the proof of these two propositions are provided in our report [31]
} 
requirements for all NCS. We proceed in two steps. First we determine the ranges of the delay requirements. Then, we adjust the delay requirements to find the ones which yield the optimal sampling rate allocation within the range.

1) Range of delay requirement determination: The lower bound $\underline{\boldsymbol{D}}=\left(\underline{D}_{h}, h \in H\right)$ of the delay range can be computed via the optimization problem of

$$
\begin{aligned}
& \underline{W}: \quad \max \sum_{h \in H} U_{h}\left(z_{h}\right) \\
& \text { s.t. } \sum_{h \in H: f \in F(l) \& f \in F(h)} z_{h} \leq \hat{c}_{l}, \forall l \in L \\
& \text { over } \hat{\boldsymbol{c}} \in \Lambda
\end{aligned}
$$

This is a simplified form of $\underline{W}$, with the $V L C M \sigma_{l}=0$ for all $l \in L$ and without the delay constraints. The solution to this problem $\underline{z}$ is the maximum achievable sampling rate considering only the network capacity constraint. This maximum achievable rate corresponds to the minimum sampling time of the NCS $T_{h}\left(\underline{z}_{h}\right)$. As our delay constraint in the original problem $W$ is that the flow delay should not exceed one sampling time, we can treat the minimum sampling time as the lower delay bound $\underline{\boldsymbol{D}}=\left(T_{h}\left(\underline{z}_{h}\right), h \in H\right)$.

Fixing the sampling rate to $\underline{z}$, we allow the maximum amount of traffic satisfying only the network capacity constraint to be injected into the network. Thus the measured delay $\overline{\boldsymbol{d}}=\left(\bar{d}_{f}, f \in F\right)$ is the upper bound of the end-to-end delay. If $\bar{d}_{f} \leq T_{h}\left(\underline{z}_{h}\right), \forall f \in F(h), \forall h \in H$, then $\underline{z}$ will also be the optimal sampling rate for the original problem $W$. If there exists $\bar{d}_{f}>T_{h}\left(\underline{z}_{h}\right)$, then we set the upper bound of the delay requirement to $\bar{D}=\overline{\boldsymbol{d}}$.

2) Optimal delay requirement adjustment: Starting from the lower bound of the delay requirement, we adjust the delay requirement of each control system based on the algorithm shown in Table I. In the algorithm, we gradually increase the delay requirement of each system $h$ from its lower bound until 1) it is smaller than the corresponding optimal sampling time based on problem $W 1$ but within a constant bound $\epsilon$; or 2) it exceeds the corresponding optimal sampling time. In the latter case, we restore the delay requirement to its last value and reduce the adjustment size from $a_{h} / m_{h}$ to $a_{h} /\left(m_{h}+1\right)$ where $m_{h}$ is initialized to 1 .

\section{Vi. Performance Evaluation}

In this section, we evaluate our cross-layer sampling rate adaptation and network scheduling algorithm using an integrated simulation tool named Networked Control System Wind-Tunnel (NCSWT) [12]. NCSWT integrates two simulators Matlab and ns-2, which allows us to simulate the control system models in Matlab/Simlink and the networking systems in ns-2. Using ns-2, a packet-level network simulator that implements all the details of the network protocol stack, we can perform highly accurate evaluation of the network effects on the NCS performance, including queueing delay and network scheduling, which is impossible by using Matlab/Simulink alone.
TABLE I

DELAY REQUIREMENT ADJUSTMENT

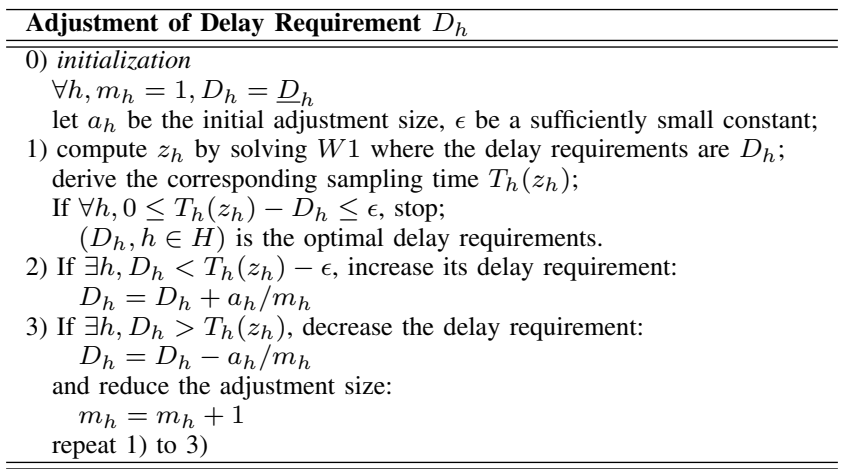

\section{A. Simulation Setup}

In our experiments, the NCS consist of three pairs of plants and controllers. Each of the three plant systems used in the experiments is the model of a single joint of a robotic arm. They are described by the continuous time state space representation as defined in (1) and (2), with the parameters $A_{p}=0, B_{p}=1, C_{p}=0.3413$. Each of the controllers is described as in (3) and (4) with $A_{c}=0, B_{c}=1, C_{c}=$ $32.1, D_{c}=8.2$. The plants and controllers are discretized based on the sampling time $T_{h}$ to obtain the discrete time equivalent. The utility function used in the experiments is the same as the function presented in Section IV. The objective is the joint velocity of each robotic arm tracks a sinusoidal reference input $r[k]=\sin (\omega k)$ for $k=0,1,2, \cdots$ with $\omega=\frac{2 \pi}{80}$. The disturbance inputs for Plant 2 and Plant 3 are white noise with the power spectral density of 1 . Plant 1 does not have any white noise input. In the wireless network, the interference range and the transmission range are set to $250 \mathrm{~m}$. The capacity of the wireless channel is $2 M b p s$. The packet size is 260 bytes. Each simulation runs for 180 seconds.

Four aspects of the system are evaluated after the first period of the reference signal when the adaptation converges: 1) the average tracking error $\bar{e}_{r r}$, which is the average absolute difference between the plant output and its reference signal. ${ }^{4}$; 2 ) the converged sampling time $T_{h} ; 3$ ) the end-to-end delay of flows associated with system $h$; 4) the channel utilization, which is the ratio of the total network load to the channel capacity.

\section{B. Simulation Results}

1) Single-hop Scenario: In the first experiment, there are six nodes in the wireless network, each hosting either a plant or a controller. All the nodes are within the transmission range of each other, forming a single-hop network topology.

Fig. 7 presents the simulation results with the optimal delay requirement derived from the delay requirement adjustment algorithm. Fig. 7(a) shows the plant outputs, and Fig. 7(b)

\footnotetext{
${ }^{4}$ The results with the optimal delay requirements are presented with the mean and its range of error based on 7 times of simulation runs.
} 

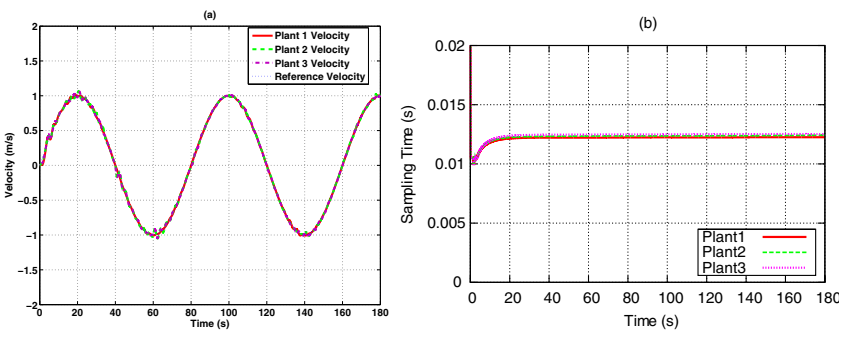

Fig. 7. Velocity Outputs with the Optimal Delay Requirement in Single-hop

illustrates the sampling time convergence of the three plantcontroller pairs. The sampling time quickly converges, and the plant outputs closely follow the reference trajectory. In Plant2 and Plant3, white noise is introduced at a period of 15 seconds, when the outputs deviate from the reference trajectory. Their gaps quickly diminish after a short period of time.
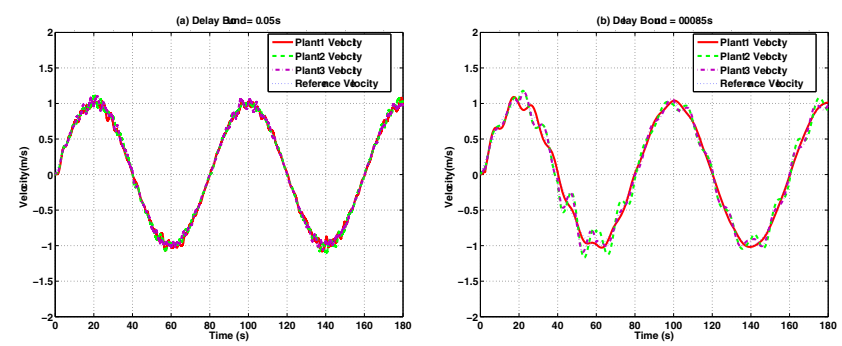

Fig. 8. Velocity Outputs with Different Delay Requirements in Single-hop

TABLE II

Performance Metrics for Different Delay Requirements

\begin{tabular}{|r|r|r|r|r|}
\hline $\begin{array}{r}\text { Delay } \\
\text { Requirements }\end{array}$ & $\begin{array}{r}\text { Average } \\
\text { Track Error }\end{array}$ & $\begin{array}{r}\text { Sampling } \\
\text { Time }\end{array}$ & $\begin{array}{r}\text { Average } \\
\text { Delay }\end{array}$ & $\begin{array}{r}\text { Channel } \\
\text { Utilization }\end{array}$ \\
\hline Optimal & $0.007 \pm 0.0043$ & 0.0125 & 0.0121 & $50 \%$ \\
\hline 0.05 & 0.0251 & 0.0087 & 0.0481 & $72 \%$ \\
\hline 0.0085 & 0.0428 & 0.2013 & 0.0087 & $3 \%$ \\
\hline
\end{tabular}

Next we compare the performance of the NCS with fixed delay requirements, which are different from the optimal one in Tab. II. Fig. 8(a) illustrates the plant outputs using the delay requirement of $0.05 \mathrm{~s}$. Fig. 8 (b) demonstrates the plant outputs using the delay requirement of $0.0085 \mathrm{~s}$. We observe that both outputs are much worse than those in Fig. 7. In addition, Plant2 and Plant3 suffer from larger oscillations than Plant1, and cannot track the reference trajectory closely, as shown in Fig. 8(b). With a larger delay requirement, the control systems are allowed to send packets with a larger sampling rate, which increases the traffic load of the networks. The average end-toend delay experienced by the control systems is more than 5 times of the sampling time. Thus, the outputs exhibit a lot of oscillation. On the other hand, a small delay requirement leads to small sampling rates, which degrade the system capability of white noise rejection. Thus, the controller cannot be notified in time about the occurrence of the white noise disturbance.

2) Multi-hop Scenario: Direct communication in wireless networks requires two nodes within the transmission range of each other. When they are out of range, intermediate nodes can provide relays to route packets. We evaluate our solution over a multi-hop wireless network with 12 nodes organized in a grid topology. The plants and controllers are deployed on nodes at the network edges. Plant 2 resides in the middle of the network. The paths of all the control system pairs are set up using the shortest-path routing algorithm.

TABLE III

Performance Metrics with Optimal Requirements

\begin{tabular}{|r|r|r|r|r|}
\hline & $\begin{array}{r}\text { Delay } \\
\text { Requirements }\end{array}$ & $\begin{array}{r}\text { Average } \\
\text { Track Error }\end{array}$ & $\begin{array}{r}\text { Sampling } \\
\text { Time }\end{array}$ & $\begin{array}{r}\text { Average } \\
\text { Delay }\end{array}$ \\
\hline Plant1 & 0.024 & $0.0020 \pm 5.705 \mathrm{e}-5$ & 0.026 & 0.031 \\
\hline Plant2 & 0.035 & $0.0186 \pm 5.797 \mathrm{e}-7$ & 0.038 & 0.048 \\
\hline Plant3 & 0.024 & $0.0159 \pm 3.549 \mathrm{e}-4$ & 0.026 & 0.023 \\
\hline
\end{tabular}

Fig. 9(a) shows the velocity outputs of the three plants with the optimal delay requirements. Tab. III compares their performance metrics. Compared with the single hop case, the plants experience larger oscillation at the beginning of the simulation. Because it takes longer time to setup the routes between the plant and controller pairs. Plant1 does not have white noise disturbance, so after convergence its velocity output follows the reference signal closely. Although Plant2 and Plant 3 have the same amount of white noise input, Plant2 has larger oscillation than Plant3. This is because the flows of Plant2 experience higher interference than those of Plant3. As a result, it has a larger sampling time and is more vulnerable to noise.

TABLE IV

Performance Metrics of the NCS with FiXed Rates

\begin{tabular}{|l|r|r|r|}
\hline & $\begin{array}{r}\text { Average } \\
\text { Track Error }\end{array}$ & $\begin{array}{r}\text { Sampling } \\
\text { Time }\end{array}$ & $\begin{array}{r}\text { Average } \\
\text { Delay }\end{array}$ \\
\hline Plant1 & 0.0132 & 0.0117 & 0.0807 \\
\hline Plant2 & 0.0223 & 0.0176 & 0.2044 \\
\hline Plant3 & 0.0139 & 0.0117 & 0.0519 \\
\hline
\end{tabular}

We further run the experiment with fixed sampling time for the three control systems. In Tab. IV, we show the average tracking error with fixed sampling time. Comparing with the errors under optimal sampling time, we observe that the three plants experience larger tracking error. Their sampling rates are about twice of the optimal rates, which leads to much longer delay in a multi-hop network. When the average delay exceeds the sampling time by orders of magnitude, the tracking error increases significantly.
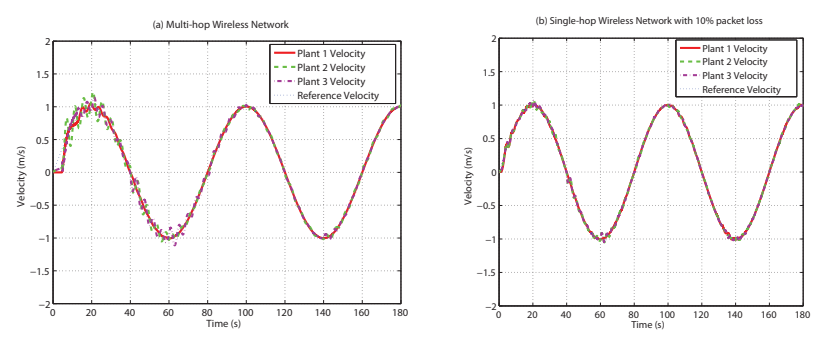

Fig. 9. Velocity outputs 
3) With wireless random packet loss: We now set up a single-hop wireless network with $10 \%$ random packet loss that may be caused by wireless interference or noise. In Fig. 9(b), we show the velocity outputs of the three plants with the optimal delay requirements. Compared with the no loss case, the plant outputs experience slightly larger oscillation, but still are able to track the reference closely.

\section{CONCLUSION}

This paper investigates the problem of NCS performance optimization in terms of tracking error minimization. It presents an optimization formulation where the objective is to maximize a utility function that characterizes the relationship between the sampling rate and the disturbance rejection capability of the control system. The constraints come from the wireless network capacity and the packet delivery delay. A distributed algorithm is presented to derive the optimal sampling rate. Our solution has desired properties from both theoretical and practical aspects. From theoretical perspective, it is shown to achieve both system stability and performance optimality. From the view of practice, it can be naturally deployed over the existing layered networking systems with well-defined cross-layer interactions. Simulation studies conducted in an integrated simulation environment consisting of Matlab/Simulink and ns-2 demonstrate that our algorithm is able to provide agile and stable sampling rate adaptation and achieve optimal NCS performance.

\section{ACKNOWLEDGEMENT}

We would like to thank our shepherd John Stankovic for his guidance and valuable inputs in helping to improve the quality of our paper. This work is supported in part by the U.S. Army Research Office (ARO W911NF-10-1-0005), the National Science Foundation (CNS-1035655, CCF-0820088) and Lockheed Martin. The views and conclusions contained herein are those of the authors and should not be interpreted as necessarily representing the official policies or endorsements, either expressed or implied, of the U.S. Government.

\section{REFERENCES}

[1] R. Gupta and M.-Y. Chow, "Networked control system: Overview and research trends," Industrial Electronics, IEEE Trans. on, vol. 57, no. 7, pp. 2527-2535, July 2010.

[2] G. Irwin, J. Colandairaj, and W. Scanlon, "An overview of wireless networks in control and monitoring," in Computational Intelligence, ser. Lecture Notes in Computer Science, D.-S. Huang, K. Li, and G. Irwin, Eds. Springer Berlin / Heidelberg, 2006, vol. 4114, pp. 1061-1072.

[3] L. Schenato, B. Sinopoli, M. Franceschetti, K. Poolla, and S. Sastry, "Foundations of control and estimation over lossy networks," Proc. of the IEEE, vol. 95, no. 1, pp. 163-187, Jan. 2007.

[4] L. Montestruque and P. Antsaklis, "Stability of model-based networked control systems with time-varying transmission times," Automatic Control, IEEE Trans. on, vol. 49, no. 9, pp. 1562-1572, Sep. 2004.

[5] M. Branicky, S. Phillips, and W. Zhang, "Stability of networked control systems: Explicit analysis of delay," in Proc. of the American Control Conference, 2000, pp. 2352-2357.

[6] M. S. Branicky, S. M. Phillips, and W. Zhang, "Scheduling and feedback co-design for networked control systems," in Decision and Control, Proc. of the 41st IEEE Conf. on, vol. 2, Dec. 2002, pp. 1211-1217.

[7] X. Liu and A. Goldsmith, "Wireless network design for distributed control," in Decision and Control, 2004. CDC. 43rd IEEE Conference on, vol. 3, 2004, pp. $2823-2829$.
[8] U. Ojha and M. Chow, "Behavioral control based adaptive bandwidth allocation in a system of unmanned ground vehicles," in IECON, Nov. 2010, pp. 3123-3128.

[9] F.-L. Lian, J. Yook, D. Tilbury, and J. Moyne, "Network architecture and communication modules for guaranteeing acceptable control and communication performance for networked multi-agent systems," Industrial Informatics, IEEE Transactions on, vol. 2, no. 1, pp. 12 - 24, feb. 2006.

[10] A. Al-Hammouri, M. Branicky, V. Liberatore, and S. Phillips, "Decentralized and dynamic bandwidth allocation in networked control systems," in IPDPS, Apr. 2006, p. 8.

[11] J. Colandairaj, G. W. Irwin, and W. G. Scanlon, "Wireless networked control systems with qos-based sampling," Control Theory Applications, IET, vol. 1, no. 1, pp. 430-438, january 2007.

[12] D. Riley, E. Eyisi, J. Bai, X. Koutsoukos, Y. Xue, and J. Sztipanovits, "Networked Control System Wind Tunnel (NCSWT) - An evaluation tool for networked multi-agent systems," in Proc. of SIMUTools, 2011.

[13] L. Bao, M. Skoglund, C. Fischione, and K. Johansson, "Rate allocation for quantized control over noisy channels," in WiOPT, International Symposium on, 2009, pp. $1-9$.

[14] Z. Li and M.-Y. Chow, "Adaptive multiple sampling rate scheduling of real-time networked supervisory control system - part ii," in IEEE Industrial Electronics, IECON 2006 - 32nd Annual Conference on, nov. 2006, pp. $4615-4620$.

[15] M. Lemmon and X. S. Hu, "Almost sure stability of networked control systems under exponentially bounded bursts of dropouts," in Proceedings of the 14th international conference on Hybrid systems: computation and control, 2011, pp. 301-310.

[16] M. Pajic, S. Sundaram, G. Pappas, and R. Mangharam, "The wireless control network: A new approach for control over networks," Automatic Control, IEEE Transactions on, vol. 56, no. 10, pp. 2305 -2318, 2011.

[17] A. Saifullah, C. Wu, P. Tiwari, Y. Xu, Y. Fu, C. Lu, and Y. Chen, "Near optimal rate selection for wireless control systems," in Proc. of RTAS, April 2012.

[18] D. Seto, J. Lehoczky, L. Sha, and K. Shin, "On task schedulability in real-time control systems," in Proc. of RTSS, 1996, pp. 13 -21.

[19] A. Kelkar and S. Joshi, "Robust control of non-passive systems via passification [for passification read passivation]," in American Control Conference, vol. 5, 1997, pp. $2657-2661$.

[20] N. Kottenstette, J. Hall, X. koutsoukos, J. Sztipanovits, and P. Antsaklis, "Design of networked control systems using passivity," IEEE Trans. Control Systems Technlology (Accepted for publication), 2012.

[21] N. Kottenstette, X. Koutsoukos, J. Hall, J. Sztipanovits, and P. Antsaklis, "Passivity-based design of wireless networked control systems for robustness to time-varying delays," in Proc. of RTSS, 2008, pp. 15-24.

[22] S. Stramigioli, C. Secchi, A. van der Schaft, and C. Fantuzzi, "A novel theory for sampled data system passivity," in IEEE/RSJ International Conference on, vol. 2, 2002, pp. 1936 - 1941.

[23] W. S. Levine, Control System Funcamentals. CRC Press, 2000.

[24] G. F. Franklin, J. D. Powel, and M. L. Workman, Digital Control of Dynamic Systems. Addison Wesley, 1997.

[25] K. Jain, J. Padhye, V. N. Padmanabhan, and L. Qiu, "Impact of interference on multi-hop wireless network performance," Wirel. Netw., vol. 11, pp. 471-487, July 2005.

[26] L. Chen, S. H. Low, M. Chiang, and J. C. Doyle, "Cross-layer congestion control, routing and scheduling design in ad hoc wireless networks," in INFOCOM, IEEE Proceedings on, april 2006, pp. 1-13.

[27] G. R. Gupta and N. B. Shroff, "Delay analysis and optimality of scheduling policies for multihop wireless networks," IEEE/ACM Trans. Netw., vol. 19, pp. 129-141, February 2011.

[28] F. P. Kelly, A. K. Maulloo, and D. K. H. Tan, "Rate control for communication networks: Shadow prices, proportional fairness and stability," The Journal of the Operational Research Society, vol. 49, no. 3, pp. 237-252, 1998.

[29] S. Low and D. Lapsley, "Optimization flow control. i. basic algorithm and convergence," Networking, IEEE/ACM Transactions on, vol. 7, no. 6 , pp. $861-874$, dec. 1999 .

[30] D. P. Bertsekas and J. N. Tsitsiklis, Parallel and distributed computation: numerical methods. Upper Saddle River, NJ, USA: Prentice-Hall, Inc., 1989.

[31] F. Qiu, J. Bai, and Y. Xue, "Optimal rate allocation in multi-hop wireless networks with delay constraints," Vanderbilt University, Tech. Rep., 2011. 